\title{
Conocimientos Sobre Prevención y Signos de Alarma del Dengue, en Padres de Escolares, Agosto 2020
}

Knowledge of Prevention and Warning Signs of Dengue Fever in Parents of Students, August 2020

\author{
Karla M. Sabillon* (D) , José Samra ** (D), Dina Álvarez *** (D) \\ *Pediatra.
}

** Coordinador Académico del Postgrado de Pediatría, Facultad de Ciencias Médicas, Universidad Nacional Autónoma de Honduras. *** Médico Pediatra, Coordinación Docencia-Investigación, Hospital María Especialidades Pediátricas.

\section{RESUMEN}

Objetivo.- Evaluar el conocimiento sobre prevención y signos de alarma del Dengue en padres o tutores de escolares que asisten al centro de educación básica gubernamental República de Costa Rica en agosto 2020.

Materiales y Métodos.- Realizamos un estudio descriptivo transversal en padres o tutores de escolares que asisten a una escuela pública gubernamental y cuentan con acceso a internet en la ciudad de Tegucigalpa. Se recopilaron datos con un cuestionario autoaplicado previamente validado de 11 preguntas que estuvo disponible en línea. Se creó una base de datos en Excel y se analizó en STATA15.

Resultados.- Se obtuvo un total de 173 participantes. La media de edad fue 35.4 años, (17-69 años). El $87.1 \%$ de los casos fueron mujeres, identificándose como principal ocupación amas de casa en $38 \%$. En cuanto al nivel educativo, el $99.5 \%$ cuentan con instrucción académica, de éstos el 26 $\%$ tienen formación universitaria. Encontramos que los padres o tutores consideran tener un adecuado nivel de conocimiento sobre dengue y medidas de prevención en $79 \%$ de los casos. Un $98 \%$ de los participantes identifican correctamente al vector y un $60 \%$ definen correctamente el cuadro clínico. El $42 \%$ identificó sangrado como principal signo de alarma de la enfermedades. Como medida de prevención más efectiva los participantes identificaron la erradicación del vector por medio de fumigación en un $53 \%$. Las principales fuentes de

Detalle de los Autores:

Karla M. Sabillon

MD. Residente del Tercer año Postgrado de Pediatría

Facultad de Ciencias Médicas.

Universidad Nacional Autónoma de Honduras. 2017-2019.

Correspondencia:

Karla Margarita Sabillon Montoya

Kmsabillon@gmail.com

+50487911705 información referida por nuestra población son a través de medios de comunicación masiva (televisión, radio, prensa escrita) en $71 \%$.

Conclusiones.- El estudio muestra que el nivel de conocimiento general de enfermedad y de signos de alarma en padres y tutores es bueno. Se identifica el cuadro clínico de dengue en fase febril, pero solo un pequeño grupo reconoce todos los signos de alarma. En cuanto a las medidas de prevención los participantes consideran que la mayor responsabilidad del control de esta infección recae en las autoridades sanitarias.

Palabras claves.- Dengue, signos de alarma, prevención, padres

\section{ABSTRACT}

Objective.- identify the knowledge, prevention, and warning signs of dengue fever in a group of parents or guardians at the Republic of Costa Rica Government Basic Education Center.

Method.- Cross-sectional observational study in 181 parents or guardians of school children from the Republic of Costa Rica Government Basic Education Center. Sampling was non-probabilistic for convenience. The survey search for the knowledge on dengue prevention and warning signs.

Results.- A total of 173 participants were obtained who met the inclusion criteria. The mean age was 35.4 years, (17-69 years). $87.1 \%$ female and $12.9 \%$ male. $0.5 \%$ of the participants are illiterate, it was found that $28.9 \%$ completed primary school, $43.9 \%$ secondary school, $26 \%$ have higher education; housewife was identified as the 
main occupation in $38 \%$ of the cases. We found that parents or guardians consider that their level of knowledge about dengue and prevention measures in $79 \%$ of the cases, and not adequate in $20 \% .98 \%$ of the participants correctly identify the vector that transmits the disease and $60 \%$ correctly define the clinical picture. $42 \%$ identified bleeding as the main warning sign of the disease, while the other warning signs are not adequately identified. As the most effective prevention measure, the participants identified the eradication of the vector by means of fumigation in $53 \%$. The main sources of information referred to by the population studied is through the mass media (television, radio, written press) in $71 \%$.

Conclusions.- The study shows that the level of general knowledge of the disease and of alarm signs in parents and guardians is good, the clinical picture of dengue is identified in the febrile phase, but only a small group recognizes all the alarm signs. Regarding prevention measures, the participants consider that the greatest responsibility for controlling this infection lies with the health authorities.

Key words.- Dengue fever, warning signs, prevention, parents

\section{Introducción}

El dengue es la arbovirosis más común afecta regiones tropicales y subtropicales, es causado por el grupo del virus de dengue que incluye 4 serotipos: DEN1, DEN2, DEN3, DEN4; y que se transmite por medio del vector Aedes aegypti. La incidencia del dengue ha aumentado en las últimas décadas, posicionandose como un problema de salud pública según datos de OPS y ocasionando una carga socioeconómica en los sistemas de salud( $1,2,3)$. Honduras es endémico para la infección por dengue, registrando casos graves desde la década de los años 70. Un estudio realizado en 2010 en pacientes pediátricos atendidos en el Hospital Escuela Universitario encontró que los grupos etarios mayormente afectados son adolescentes y escolares (4).

Para reducir la morbimortalidad de la enfermedad se requieren estrategias multimodales que involucran la participación gubernamental, población civil y sistemas de salud. Son muchas las políticas adoptadas por autoridades de salud para disminuir el impacto de la enfermedad. La comunidad adquiere un rol determinante y activo en el control del vector siendo entonces una responsabilidad conjunta entre el Estado y la población $(4,5)$. Los habitantes también tiene un rol fundamental en el reconocimiento de signos tempranos y asistir prontamente a los centros asistenciales. Los pacientes y padres o tutores en caso de población pediátrica tienen que identificar signos de alarma, evitar automedicación y acudir al hospital prontamente (6). El Estado por su parte debe garantizar que exista estructura de atención accesible a la población (centros de salud, recursos humanos, insumos, etc) y el equipo sanitario debe aplicar guías de manejo actualizadas enfocadas en diagnóstico temprano, tratamiento oportuno y prevención de complicaciones. La Secretaria de Salud de Honduras ha diseñado campañas nacionales difundidas a través de prensa escrita, radio, televisión, redes sociales, encaminadas a educar a la población. Así mismo promueven campañas de educación continua en escuelas, centros de salud, hospitales y distribuyen folletos informativos a nivel nacional. La evaluación constante de los programas de prevención y el nivel de conocimiento que la población tiene de ellos permite adoptar mejoras que generen un impacto positivo en la salud pública $(7,8)$. Bajo este marco se plantea la necesidad de evaluar el nivel de conocimiento sobre prevención y signos de alarma del dengue en padres o tutores de escolares.

\section{Materiales y Método}

Tipo de estudio: se realizó un estudio observacional, descriptivo en padres o tutores del centro básico de educación gubernamental República de Costa Rica, en la ciudad de Tegucigalpa. La elección del centro educativo fue al azar. Este centro brinda atención a niños desde primer a sexto grado de primaria. Con la situación ocasionada por la pandemia por SARS-CoV2 los estudiantes reciben sus clases de forma virtual. 
El universo fueron 200 padres o tutores de niños escolares que tiene acceso a internet. Se calculó una muestra de 177 participantes para un IC 99\%. Se tomó como criterio de inclusión: ser padre o tutor de un estudiante de esta escuela, acceso a internet, firma de consentimiento informado de participación voluntaria en el estudio (electrónica). Se excluyeron docentes del centro y menores a 16 años.

\section{Procedimiento}

La recolección de datos fue mediante la aplicación de un cuestionario estructurado, adaptado, disponible en línea, ya validado, obtenido de "Validación de un instrumento para medir el nivel de conocimientos en pobladores sobre dengue, signos de alarma y prevención, en un distrito de reciente brote de dengue para recolección de datos" publicado en 2018 que alcanzó un coeficiente de Kuder-Richardson de 0.8 y coeficiente de Alfa de Cronbach mayor a 0.82; consta de 11 preguntas y 34 ítems divididas en 8 preguntas de generalidades, 3 de conocimientos de la enfermedad, 1 de signos de alarma, 7 de prevención.

La encuesta se compartió en línea con el apoyo del docente de la Institución, de autoaplicación y con una duración de 15 minutos en promedio; se informó a los padres la disponibilidad de la encuesta en línea y se mantuvo habilitada durante 10 días del 17 al 25 agosto. Haciendo recordatorio el día 4 y día 8 . Una vez enviada la encuesta no se podía reingresar al sitio online. Se distribuyó el instrumento de recolección de datos mediante el enlace para Google Forms, registrado en una base Microsoft Excel y analizada en programa STATA15. Las variables categóricas se expresan como frecuencias y porcentajes y las variables continuas con medidas de tendencia central.

\section{Consideraciones Éticas}

Se contó con la aprobación del Comité de Ética en Investigación del Hospital María, Especialidades Pediátricas, con número de registro: IRB00011269; además se contactó con las autoridades del centro de educación básica quienes dieron aprobación para el estudio y la colaboración de los docentes para difundir el instrumento de recolección de datos. La información fue confidencial, no se incluyeron datos personales.

\section{Resultados}

Se recolectaron un total de 181 participantes, para el análisis se incluyeron 173 casos que cumplen criterios de inclusión.

Las respuestas al cuestionario se obtuvieron en dos picos el $71 \%$ de los datos se recogió al 4 día (primer recordatorio) y el $17 \%$ el día 8 (segundo recordatorio). Se obtuvo participación de padres o tutores de todos los grados distribuidos así: $13.3 \%$ primer grado; $16.7 \%$ segundo grado, $13.8 \%$ tercer grado, $14.5 \%$ cuarto grado, $17.38 \%$ quinto grado y $24.2 \%$ sexto grado.

Dentro de las características de la población la media de edad fue de 35.4 años, (Min 17- Max 69 años). El $87.1 \%$ eran femeninos. En cuanto al nivel educativo $0.5 \%$ son analfabetas y $99.5 \%$ tienen la siguiente formación académica: $28.9 \%$ primaria, $43.9 \%$ secundaria y $26 \%$ tienen educación Superior. Dentro de las actividades $38 \%$ son amas de casa, $24.27 \%$ realizan actividades de trabajo independiente, . $24.7 \%$ son empleados trabajo formal, $4 \%$ trabajan en área de salud, $4.6 \%$ son estudiantes. Dentro de las condiciones de vivienda se evalúa el acceso que los participantes tienen a agua potable, encontramos que el $94.2 \%$ recibe agua del SANAA, $2.3 \%$ de camión cisterna, y 1.2\% usa sistema privado y público. El $71.7 \%$ almacena agua, sen baldes o botes (52\%), tanques 0 cisternas $42 \%$, y $4 \%$ en pilas.

\section{Preguntas de conocimiento de padres o tutores acerca de dengue.}

Ante la pregunta: En su opinión, ¿Cuál es el nivel de conocimientos que usted posee sobre el dengue? El padre o tutor se autoevalúa desde muy bajo (poco conocimiento) a muy bueno (buen nivel de conocimiento). Encontramos que los padres o tutores consideran que su nivel de conocimiento es muy bueno 62 (36\%); bueno 75 $(43 \%)$, regular $31(18 \%)$, bajo $1(0.6 \%)$ o muy bajo $2(1.2 \%), 2$ participantes no contestaron. El $86 \%$ de los padres con educación universitaria consideran tener un nivel de conocimiento adecuado sobre dengue, disminuyendo a $81 \%$ en padres con educación media y $28 \%$ en padres con educación primaria. 
Se indagó sobre el agente causal del dengue, dando diferentes opciones de respuesta, con una única respuesta correcta.

El $55 \%$ de los participantes contestaron que el agente causal es un virus, y el $98 \%$ tiene seguridad en que se transmite por un vector mosquito Aedes Aegypti. Se les consulto donde se reproduce el zancudo que trasmite el dengue contestando $49 \%$ en agua limpia mientras que $46 \%$ piensa que en agua sucia.

Gráfico $\mathbf{N}^{\mathbf{0} 1}$. Distribución porcentual sobre conocimiento de agente causal, modo de transmisión y sitio de reproducción del vector causante de dengue.

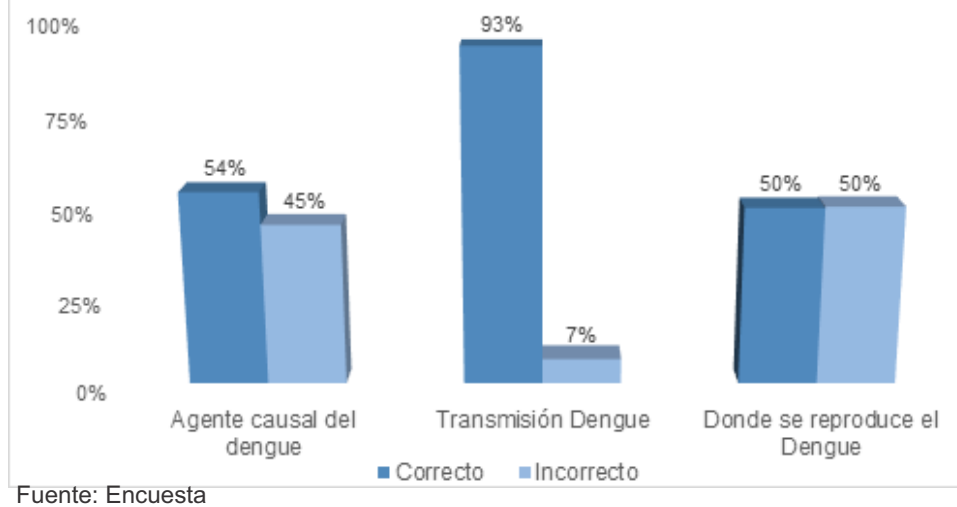

Se identifica cuadro fiebre como manifestación clínica mandatoria en dengue ( $n=169 ; 98 \%)$, y asociada a otros síntomas como cefalea, mialgias, artralgias $59 \%$ de los casos; fiebre asociada a mialgia, artralgias y nauseas en un $26 \%$ de los casos, y fiebre asociada a secreción nasal en un $14.5 \%$ de los casos.

Tabla No 1. Identificación de manifestaciones clínicas y signos de alarma en dengue .

\begin{tabular}{|c|c|c|}
\hline \multirow[b]{2}{*}{ Nivel conocimiento Dengue $n=171$} & Sí & No \\
\hline & $n(\%)$ & $\mathrm{n}(\%)$ \\
\hline $\begin{array}{l}\text { Manifestaciones clínicas } \\
\text { Fiebre asociada con cefalea, } \\
\text { mialgias, artralgias } \\
\text { Fiebre asociada con mialgias, } \\
\text { artralgias, nauseas } \\
\text { Fiebre asociada con rinorrea, mialgias }\end{array}$ & $\begin{array}{l}103(59.5 \%) \\
45(26.3 \%) \\
25(14.6 \%)\end{array}$ & $\begin{array}{l}68(38.7 \%) \\
126(11.4 \%) \\
146(85.4 \%)\end{array}$ \\
\hline \multirow{2}{*}{ Signos de Alarma } & Unico & Acompañado \\
\hline & $\mathrm{n}(\%)$ & $\mathrm{n}(\%)$ \\
\hline $\begin{array}{l}\text { Sangrado } \\
\text { Vómito } \\
\text { Dolor abdominal } \\
\text { Otros }\end{array}$ & \begin{tabular}{|l}
$72(42.1 \%)$ \\
$31(18 \%)$ \\
$29(17 \%)$ \\
$10(6 \%)$
\end{tabular} & $\begin{array}{l}99(57.89 \%) \\
140(81.8 \%) \\
142(83 \%) \\
161(94 \%)\end{array}$ \\
\hline
\end{tabular}

Fuente: Encuesta
Los padres y/o tutores reconocen el sangrado como principal signo de alarma en un $42 \%$ de los casos, vómitos en $18 \%$, dolor abdominal en el $17 \%$ de los casos y edema o lipotimia en un $6 \%$ de los casos respectivamente.

Gráfico № 2: Identificación de signos de alarma Signos de alarma Dengue identificados por los padres

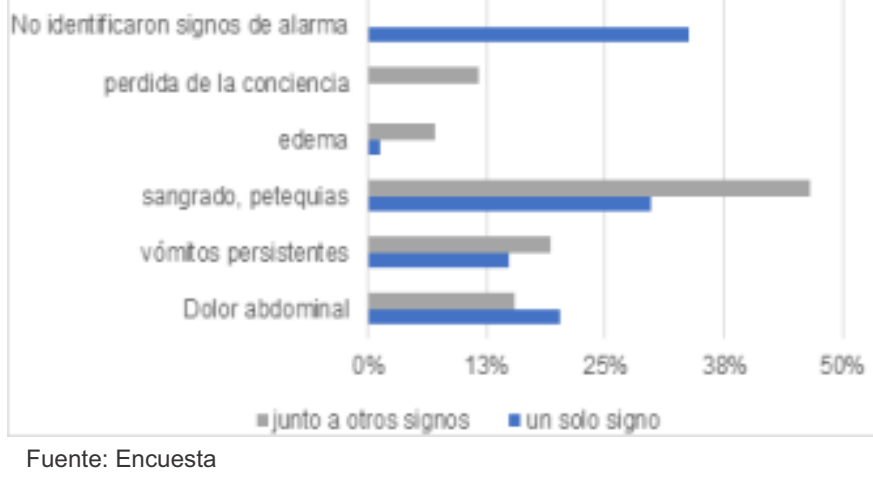

\section{Medidas de prevención}

Los encuestados consideran que la fumigación (53\%) es el principal método para prevenir o evitar la picadura por el vector.

Tabla № 2 Al preguntarles por medidas que conocen para eliminar los criaderos de zancudo o mosquitos del dengue consideran que lavando depósitos de agua (88.3\%), eliminando agua acumulada $(85.6 \%)$ y manteniendo tapados los depósitos con agua (78.9\%) son las medidas efectivas. El $95 \%$ de la población encuestada considera que el abate sirve para matar larvas de zancudos.

\begin{tabular}{|l|c|c|}
\hline Variable & Frecuencia & Porcentaje \\
\hline ¿Qué medidas conoce Ud. para & & \\
eliminar los criaderos del zancudo & & \\
o mosquitos del dengue? & & \\
Eliminando la basura & 99 & $55 \%$ \\
Lavando depósitos & 159 & $88.3 \%$ \\
Tirando objectos cerca a su casa & 63 & $35 \%$ \\
Eliminando agua acumulada & 154 & $85.6 \%$ \\
Manteniendo tapados los depósitos & 142 & $78.9 \%$ \\
con agua & 0 & $0 \%$ \\
No sabe & \multicolumn{2}{|l|}{} \\
\hline Para qué sirve el abate & 7 & $4 \%$ \\
Incorrecto & 164 & $95 \%$ \\
Correcto: Matar las larvas del zancudo & 2 & $1 \%$ \\
No lo conozco/ sin respuesta & \\
\hline Fuente: Encuesta &
\end{tabular}


encuestados almacena agua dentro de su vivienda y lo almacenan en baldes, botes (52\%) y tanques, cisterna (42\%), solo $4 \%$ en pilas. La población encuestada refiere que se debe lavar los depósitos de agua una vez a la semana (45\%).

\section{Principales fuentes de educación sobre dengue de los padres o tutores de centro de educación básica}

La información sobre dengue que tienen los participantes del estudio proviene principalmente de medios de comunicación tradicionales como televisión, radio, prensa escrita. (71\%); además de charlas educativas por personal de salud (18\%); un participante informó no haber recibido información sobre la enfermedad. Ver gráfico N. 3

Gráfico 3: Fuentes de educación sobre dengue referidas por padres o tutoresiba.

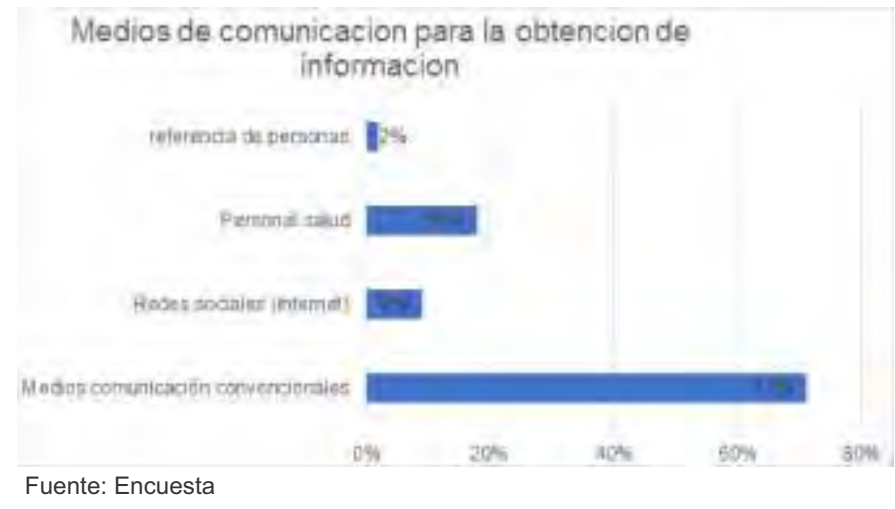

\section{Discusión}

La Estrategia Integral de Atención (EIA) contra dengue, vigente en Honduras desde el 2003, ha propuesto estudios similares al actual, determinar los conocimientos de una población sobre aspectos de salud pública en enfermedades vectoriales. Llama la atención como la mayoría de los encuestados son del sexo femenino (8 de cada 10). Esto nos pone de manifiesto el rol de la mujer en el hogar en nuestro país, como ama de casa o con un trabajo remunerado con una educación superior a la básica. En la actualidad, al menos seis de cada diez mujeres latinoamericanas participan en el mercado laboral (9).

Los resultados de esta investigación ponen en evidencia que aun cuando el dengue se ha encontrado endémico en nuestro país desde hace 20 años, la percepción de conocimientos sobre dengue que los entrevistados tienen es considerado por ellos entre bueno (42.9\%) y muy bueno (37.9\%), las respuestas a la encuesta no fueron correctas. Los encuestados contestaron acertadamente el vector similar a lo obtenido en estudios equivalentes internacionales $(10,11)$. Mientras que poco más de la mitad conoce que la enfermedad la produce un virus. Esto muestra un desconocimiento de la etiología (55\%) pero mayor a lo encontrado en un estudio similar en Colombia donde solo el $7 \%$ identifico causa (12). La sintomatología típica del cuadro no fue identificada por la mayoría, considerando que el único signo de alarma que era conocido fue el sangrado mientras que otros signos de alarma dados por la OMS menos del $20 \%$ los pudieron identificar. Llama la atención por el que signo de alarma más notorio y recurrente es el dolor abdominal(13).

Estas cifras denotan la poca identificación de los signos de alarma, incluso en aquellos con nivelo. El cuadro que describe la OMS en la fase febril aguda dura de 2 a 7 días y suele acompañarse de enrojecimiento facial, eritema, dolor corporal generalizado, mialgias, artralgias, cefalea y dolor retro orbitario (14). Los encuestados consideraron que la fiebre principal y asociado al cuadro característico un $60 \%$ dolor de cabeza, dolor muscular y de articulaciones son el cuadro más característico. Un pequeño parte 15\% entrevistados creen que los síntomas de un cuadro catarral Is son posibles en la enfermedad. Otro estudio más reciente realizado en Colombia reporta que menos del $40 \%$ reconocen otros síntomas diferentes a la fiebre (12). En nuestra muestra existe una mejor identificación de los síntomas del dengue.

Los participantes del estudio son ciudadanos que viven en el casco urbano. Siendo estas colonias del casco urbano de alto nivel de positividad vectorial (Índice de Infestación) y de transmisibilidad de la enfermedad en la ciudad capital (15). La investigación refleja que la mayoría de los hogares a los que pertenecen los encuestados reciban agua potable de SANAA. La crisis de agua que se vive en Tegucigalpa durante gran parte del año y sobre todo en verano. Esto ha obligado a drásticos racionamientos. Habitantes reciben agua potable durante el verano por horas cada 5 a 9 días y en casos extremos lugares que pueden recibirla cada más de diez días(16). La mayoría afirma almacenar agua en baldes y pilas, muchas 
veces dentro de sus viviendas, incrementando el riesgo de propagación de la enfermedad por mayor cantidad de zancudos.

Los participantes de la encuesta se mantienen informados sobre el dengue por medio de la televisión en $56 \%$ y un $19 \%$ a través del personal de salud. Obteniendo similares resultados en estudio realizado en Indonesia en 2018 (17) mientras que en un estudio en Vietnam siempre predominio la televisión, pero también usaban las redes sociales para su información sobre dengue(18). Existe desinformación sobre donde se reproduce el mosquito trasmisor del dengue al contestar aguas sucias un $46 \%$ o incluso basuras un $4 \%$ considerando que solo mitad contesto correctamente que se reproduce en agua limpia. Siendo esto de crucial importancia ya que la mayoría $(72 \%)$ de los encuestados almacenan agua dentro de su vivienda en baldes, botes (53\%) y tanques y cisternas (42\%). Aún más alarmante es que $46.3 \%$ opina que debe lavar donde almacena el agua para consumo una vez a la semana considerando que las larvas del zancudo Aedes aegypti necesitan menos de una semana para alcanzar su vida adulta (19). Para eliminar los criaderos de zancudos la mayoría de los entrevistados consideró que, lavando depósitos, eliminado agua acumulada y manteniendo tapados los depósitos con agua es la solución.

En las medidas de prevención del dengue la población encuestada contesto similar a la población internacional en su mayoría respondieron la fumigación (93.9\%) y el uso de repelente $(75.4 \%)$ son métodos para la prevención efectivos. Sin embargo, una parte de los entrevistados que piensan que existe la vacunación contra el dengue en Honduras $(17.3 \%)$. Casi nadie $(0.6 \%)$ consideró que, limpiando los criaderos de zancudos, o las llantas con agua estacada, las pilas, drones o recipientes, tapar recipientes o lavar con cloro y detergente las pilas podría evitar la picadura del zancudo. Las medidas de prevención sobre el dengue tienen resultado similares un estudio realizado en el 2015 en población rural del sur de Honduras (20) y en la literatura latinoamericana $(21,22)$ distinto a la literatura encontrada en Asia donde los encuestados consideraron que la medida para evitar el dengue era evitando agua estancadas y la limpieza $(11,17,23)$. La limitación del estudio el no identificar las carac- terísticas del servicio del SANAA en el hogar de los encuestados y el no identificar directamente por qué se almacena agua en su hogar.

\section{Conclusiones}

1. El análisis muestra que los encuestados en su mayoría son amas de casa quienes alcanzaron una educación mayor o igual a sexto grado. Esto es razonable considerando que la cultura local asigna a la madre el apoyo en el hogar y a los hijos. Por lo tanto, este sector debe ser tomado en cuenta para mayor educación acerca del dengue.

2. La población encuestada logra identificar la definición de caso de dengue de la Secretaría de Salud, pero no identifica los signos de alarma predictores morbimortalidad.

3. La principal fuente de información son medios de comunicación convencionales (televisión, radio y prensa) pero hay aumento del uso de redes sociales. Los medios de comunicación deben ser explotados por las fuentes oficiales de salud para garantizar que la información sea la adecuada en la prevención del dengue y sus complicaciones.

\section{Recomendaciones}

1. A la Secretaría de Salud, la creación de un comité técnico científico para supervisar el contenido de los medios de comunicación para garantizar información apropiada y útil acerca de factores de riesgo, identificación de caso y de signos de complicaciones del dengue para poder prevenir y tratar oportunamente la enfermedad.

2. A los medios de comunicación, la creación de espacios permanentes para la educación continua de la población, ya que la repetición de la información es clave para afianzar conceptos y lograr un aprendizaje efectivo.

3. A la Secretaría de Educación, crear un sistema de medición del conocimiento de conceptos del dengue para los padres de familia estandarizado para todos los centros educativos del país. Esta moción podría iniciarse mediante la creación de centros piloto apoyados por entes internacionales que ayuden a financiar y gestionar dicho sistema de evaluación.

Conflicto de interés.- ninguno 


\section{Bibliografía}

1. Avila-Aguero ML, Camacho-Badilla K, Brea-DelCastillo J, Lizbeth C, Dueñas L, Luque M, et al. Epidemiología del dengue en Centroamérica y República Dominicana. Revista Chilena de Infetología. 2019 Diciembre; 36(6).

2. Khetarpal N, Khanna I. Review Article: Dengue Fever: Causes, Complications, and Vaccine Strategies. Journal of Immunology Research. 2016 Junio; 2016: 14.

3. Wilder-Smith A, Ooi EE, Horstick O, Wills B. Dengue. Lancet. 2019 Enero; 393: 350-363.

4. S RZ, L Z, M. S. Caracterización clínica y hematológica de pacientes pediátricos con Dengue en Honduras. Rev Medica Risaralda. 2014;20(2): 95-100.

5. Honduras SdS. Gobierno de la Republica de Honduras: Secretaria de Salud. [Online].; 2020 [cited 2020 Octubre 12. Available from: http://www.salud.gob.hn/site/index.php/compone nt/k2/itemlist/category/4-dengue-chikv-zika.

6. Mendoza-Cano O, Hernandez-Suarez CM, Trujillo $X$, Ochoa Diaz-Lopez H, Lugo-Radillo A, Espinoza-Gomez F, et al. Cost-Effectiveness of the Strategies to Reduce the Incidence of Dengue in Colima, Mexico. International Journal of Environmental Research and Public Health. 2017 June; 14(890): 1-9.

7. OPS Honduras. PAHO.ORG. [Online].; 2019 [cited 2020 Octubre 12. Available from: https:// www.paho.org/hon/index.php?option=com conte nt\&view=article\&id=958:lanzamiento-estrategiaintegrada-prevencion-control-denguehonduras\&ltemid=229.

8. Organizacion Panamericana de la Salud. Estrategia de Gestión Integrada para la prevención y control del dengue en la Región de las Américas. In OPS; 2017; Washington D.C. p. 23.

9. PNUD America Latina y el Caribe. [Programa de Naciones Unidades para el Desarollo].; 2019 [cited 2020 Octubre 12. Available from: https://www. lati namerica.undp.org/content/rblac/es/home/pressc enter/pressreleases/2019/acelerar-la-participa cion-laboral-femenina-es-crucial-para-la-im.html.

10. Gutierrez C, Montenegro-Idrogo JJ. Conocimiento sobre dengue en una región endemica de Peru. Estudio de base poblacional. Acta Medica Peruana. 2017 Diciembre; 34(4): 283-288.

11. Kumaran E, Duom D, Keo V, Sokha L, Sam B, Chan V, et al. Dengue knowledge, attitudes and practices and their impact on community-based vector control in rural Cambodia. Plos Neglected Tropical Diseases. 2018 Febrero; 12(2): 1-16.

12. Benitez- Diaz L, Diaz- Quijano FA, Martinez- Vega RA. Experiencia y percepcion del riesgo asociados a conocimientos, actitudes y practicas sobre dengue en Riohacha, Colombia. Ciencia \& Saude Colectiva. 2020 Marzo; 25(3).

13. Izquierdo EstevezA, Martinez Torres E. Utilidad de la identificacion de los signos de alarma en niños y adolescentes con dengue. Revista Cubana de Pediatria. 2019;91(2): 1-13.

14. Organizacion Panamerica de la Salud. Guías para la atención de enfermos en la región de las américas. Biblioteca Sede de la OPS. 2015; 2: 125-126.

15. Secretaria de Salud. Situación Epidemiologica de Dengue hasta la Semana Epidemiologica segun datos recolectados por alerta respuesta. Reporte. Tegucigalpa: Gobierno de la Republica de Honduras, Unidad de Vigilancia de la Salud; 2020.

16. EV. Población capitalina es agobiada por la falta de agua. La Tribuna. 2019 Septiembre.

17. Harapan H, Rajamoorthy Y, Anwar S, Bustamam A. Knowledge, attitude, and practice regarding dengue virus infection among inhabitants of Aceh, Indonesia: a cross sectional study. BMC Infectious Diseases. 2018 Febrero; 18(96): 1-16.

18.TQ V, Pham TT P. Revisiting dengue-related knowledge, attitudes and practices: a crosssectional study in Ho Chi Minh City, Vietnam, 2018. The Journal of the Pakistan Medical Association. 2019 Mayo; 69(6): S108-S117.

19.Vannavong N, Seidu R, Stenstrom TA, Dada N, Overgaard J. Effects of socio-demographic characteristics and household water management on Aedes Aegypti production in suburban and rural villages in Laos and Thailand. Parasites \& Vectors. 2017Abril; 10(170): 1-14.

20.Del Cid Morazan AF, Barcan M, Gonzalez H, Barahona Andrade DS. Conocimientos, actitudes y practicas sobre las arbovirosis. iMedPub Journals. 2017; 13(1): 1-5.

21.Cabrera R, Gomez de la Torre- Del Carpio A, Bocanegra Jesus Al. Conocimientos, actitudes y practicas sobre dengue en estudiantes de educaicon primaria en Chorrillos, Lima, Perú. Anales de la facultad de Medicina. 2016 Junio; 77(2): 129-135.

22.Diaz-Quijano FA, Martinez-Vega RA, RodriguezMorales J. Association between the level of education and knowledge attitudes and practices regarding dengue in the Caribbean region of Colombia. BMC Public Health. 2018 Enero; 18(143).

23.Xu JW, Lui H, Ai D, Yu Y, Yu B. The Shan people's health beliefs, knowledge and perceptions of dengue in Eastern Shan Special Region IV, Myanmar. Plos Neglected Tropical Diseases. 2019 Junio; 13(6).

24. Rather IA, Parray HA, Lone JB, Paek WK, Jeongheui L, Bajpai VK, et al. Prevention and Control Strategies to Counter Dengue Virus Infection. Frontiers in Cellular and Infection Microbiology. 2017 Julio; 7: 2-8.

25.Davila-Gonzales JA, Guevara-Cruz LA, PeñaSanchez ER, Diaz-Velez C. Validacion de un instrumento para medir el nivel de conocimeintos en pobladores sobre dengue, signos de alarma y prevencion en un dsitrito de reciente brote de dengue. Revista Hispanoamericana de Ciencias de la Salud. 2018 Septiembre; 4(3): 119-128. 